\title{
Three-dimensional flow structures and dynamics of turbulent thermal convection in a cylindrical cell
}

\author{
Chao Sun and Ke-Qing Xia \\ Department of Physics, The Chinese University of Hong Kong, Shain, Hong Kong, China \\ P. Tong \\ Department of Physics, Hong Kong University of Science and Technology, Clear Water Bay, Kowloon, Hong Kong, China
}

(Received 24 March 2005; published 12 August 2005)

\begin{abstract}
The technique of particle image velocimetry is used to study the velocity field of turbulent Rayleigh-Bénard convection in an aspect-ratio-1 cylindrical cell filled with water. By measuring the two-dimensional (2D) velocity vector map in defferent vertical cross sections of the cell, we investigate the 3D structures and dynamics of turbulent thermal convection. The experiment reveals how thermal plumes synchronize their emissions and organize their motions spatially between the top and bottom plates, leading to an oscillatory motion in the bulk region of the fluid with a period equal to twice the plume's cell-crossing time. From the measured instantaneous velocity vector map, we find the phase relationship between the velocity components along different directions and at different positions in a 2D plane. These phase relations illustrate how the convecting fluid in different regions of the cell interact with each other and generate a synchronized and coherent motion in a closed system.
\end{abstract}

DOI: 10.1103/PhysRevE.72.026302

PACS number(s): 47.27. $-\mathrm{i}, 05.65 .+\mathrm{b}, 44.25 .+\mathrm{f}$

\section{INTRODUCTION}

Because of its connection to many convection phenomena occurring in nature, such as the atmospheric and mantle convections, turbulent Rayleigh-Bénard convection continues to attract intensive research efforts in recent years. It is also an excellent model system to study spatial organization and temporal synchronization of coherent structures in a closed cell. In turbulent Rayleigh-Bénard convection, the flow is initiated and maintained by the buoyancy force produced by an applied temperature difference across the height of the convection cell. The dynamics of the flow is determined by the geometry of the cell and two dimensionless parameters: the Rayleigh number $\mathrm{Ra}=\alpha g \Delta T L^{3} /(\nu \kappa)$ and the Prandtl number $\operatorname{Pr}=\nu / \kappa$, where $\Delta T$ is the applied temperature difference, $L$ is the height of the cell, $g$ is the acceleration due to gravity, and $\alpha, v$, and $\kappa$ are, respectively, the volume expansion coefficient, kinematic viscosity, and thermal diffusivity of the fluid.

At sufficiently high values of Ra, a large-scale circulation (LSC) is formed across the height of the convection cell $[1,2]$. This large-scale circulation, also known as the "wind" in turbulent convection, has been studied extensively in recent years. Many experimental, theoretical, and numerical investigations have been carried out to study various aspects of the LSC, such as the mean velocity profile in the LSC plane, the boundary layer thickness and its scaling with Ra and Pr, the geometry effect of the convection cell on the spatial structure of the LSC and on the velocity statistics over varying $\mathrm{Ra}$, and the statistical properties of random reversals of the rotation direction of the LSC [3-21]. Among the experimental studies, Qiu and Tong [14] conducted a systematic investigation of the flow structure in an aspectratio- 1 cylindrical cell filled with water. Using the technique of laser Doppler velocimetry (LDV), they measured the ve- locity profiles in the plane of the LSC and demonstrated that the mean flow pattern in the bulk region of the cell indeed has a flywheel structure, which was first proposed by Kadanoff [22,23] and Zocchi et al. [24]. Their experiment also revealed that the warm and cold plumes are separated laterally in the two opposing sidewall regions, which exert buoyancy forces on the bulk fluid and drive the LSC. A recent visualization experiment by $\mathrm{Xi}$ et al. [25] studied the onset of the LSC and confirmed that the thermal plumes are indeed responsible for the formation and sustenance of the LSC.

The flywheel structure for the velocity field in the LSC plane is a time-averaged result, and thus it is a simplified model without including the dynamics of the flow field in the closed cell. An intriguing feature of the convection dynamics is the emergence of a well-defined low-frequency oscillation, which was observed in both the temperature $[2,26]$ and velocity [27-29] fields. This oscillation takes place in the hard turbulent regime when the Rayleigh number becomes larger than a critical value $\operatorname{Ra}_{c}\left(\sim 5 \times 10^{7}\right)$. To understand the temperature oscillation in turbulent convection, Villermaux [30] proposed that this oscillation is caused by a thermal boundary layer instability triggered by the arriving thermal plumes, which are transported along the cell periphery by the largescale circulation. According to this model, the emission of warm (or cold) plumes near one of the conducting plates is triggered by the arriving cold (warm) plumes from the opposite conducting plate. The emitted plumes in turn travel to the opposite conducting plate to trigger the emission of thermal plumes there. This alternating emission of warm and cold plumes between the two conducting plates takes place in a time interval $t_{0}$, which is the time required for the plume to transverse the height of the cell. As a result, the overall temperature oscillation period is given by $\tau_{0} \simeq 2 t_{0}$.

To test Villermaux's model, Qiu and Tong [31,32] measured the temperature cross-correlation functions between 
two positions located at the two opposite sidewall regions. Their experiment confirmed the physical picture in Villermaux's model that the thermal plumes are indeed emitted alternately between the two conducting plates with a time interval approximately equal to the plume's cell crossing time $t_{0}$. In a more recent experiment, Qiu et al. [33] carried out a systematic study of the velocity oscillation in turbulent convection. From the simultaneous measurements of the local velocity and temperature, they obtained the temperaturevelocity correlation functions and found that the temperature and velocity oscillations are driven by a common mechanism. Because the warm and cold plumes are separated laterally in the two opposing sidewall regions and exert buoyancy forces on the bulk fluid, an alternating emission of the warm and cold plumes gives rise to a periodic impulsive torque, which drives the large-scale circulation continuously. The velocity oscillations observed in different directions are simply the response of the bulk fluid to the periodic thermal forcing in the closed cell.

While the above temperature and velocity measurements have provided an interesting picture about how the thermal plumes organize themselves in a closed cell and generate a large-scale flow structure, which rotates and oscillates coherently in a turbulent environment, there are still several important issues that need to be further studied. First, our current knowledge about the LSC is confined mainly in the plane of the LSC. This is partially due to the fact that the LDV technique, which was used to obtain information about the velocity field, is a local probe that can only measure the local velocity at a single point at a given time. It is difficult experimentally to obtain three-dimensional information about the velocity field using a local probe. Second, our current understanding of the large-scale motion in turbulent convection is focused mainly on the structure and dynamics in the bulk region of the flow, in which cell boundaries have less influence. To study the dynamics near the thermal boundary layers, from which the thermal plumes are emitted, and its interaction with the large-scale flow in the bulk region, one needs to measure the two-dimensional (2D) velocity field in different planes.

Recently, Funfschilling and Ahlers [34] used a shadowgraphic method to study the motion of thermal plumes in a horizontal plane near the upper and lower conducting plates. They found that the thermal plumes appear as sheetlike objects when viewed from the top and their motion undergoes a periodic oscillation in the horizontal plane parallel to the conducting plate. The oscillation frequency observed near the thermal boundary layer is the same as that measured in the bulk fluid region. In a different experiment [35], Haramina and Tilgner used a dye labeling technique and found that there exists a coherent structure in the thermal boundary layer, which manifests as dye-enriched streaks along the mean flow direction. These streaks do not look like the sheetlike structures observed in the shadowgraphic study [34] and it was suggested that the dye-enriched streaks may be manifestations of the presence of counter-rotating vortex pairs. These experiments indicate that the velocity field in a closed convection cell may have more complicated threedimensional fine structures and a further study of threedimensional flow structures and dynamics in turbulent convection is needed.
The technique of particle image velocimetry (PIV) provides us a convenient tool to directly visualize and measure the three-dimensional flow field in a particular plane of interest [36]. With the PIV technique one captures two consecutive two-dimensional images of the seed particles, using a charge-coupled device (CCD) camera situated normal to an illuminating light sheet. Spatial correlations between the two images are then calculated to obtain information about the displacement of each particle, from which one obtains the velocity map. The main advantage of the PIV method is its ability to follow the motion of a 2D flow field. With the 2D time series data, one can obtain both the time-averaged and the dynamic properties of the 2D flow field. By measuring the velocity maps in different planes, one can examine the 3D structures and dynamics of a velocity field with a shorter period of experimental time. In a recent experiment [37], Xia et al. used the technique of PIV to study the flow structure of turbulent convection in a rectangular box. It was shown that the PIV technique is indeed a powerful tool for direct visualization of the flow field in turbulent convection. Because there are no temperature and velocity oscillations in this geometry,[37] the flow dynamics in the rectangular cell is quite different from that in the cylindrical cell. It appears that the cylindrical geometry has produced a rich array of interesting features of turbulent convection in terms of temporal synchronization and spatial organization of plume motion and the associated flow modes. Therefore, the cylindrical convection system has become an ideal system to study the dynamics of coherent structures in turbulent convection.

In this paper, we focus our attention on the threedimensional flow structure and dynamics of turbulent thermal convection in an aspect-ratio-1 cylindrical cell. In particular, we want to understand the following three unresolved issues in turbulent convection: (1) the 3D structure of the mean velocity field in the cylindrical cell; (2) synchronized motion of thermal plumes between the upper and lower conducting plates; and (3) spatial and temporal coherence of bulk velocity oscillations. To address these issues, we use the technique of PIV to measure the $2 \mathrm{D}$ velocity maps in different cross sections of the cell and over an extended period of time. These PIV measurements allow us to reconstruct the 3D structure and dynamics of the convective flow in the bulk region and figure out the overall driving mechanism of the convection system. The remaining part of this paper is organized as follows. Sec. II contains a brief description of the experimental method and apparatus. In Sec. III we present the experimental results, which are divided into three parts: (A) three-dimensional structure of the mean velocity field, (B) synchronized motion of thermal plumes between the top and bottom plates, and (C) spatial coherence of the bulk velocity oscillations. Finally, we summarize our findings in Sec. IV.

\section{EXPERIMENT}

\section{A. Convection cell}

The experiment is conducted in a vertical cylindrical cell filled with water. The diameter and height of the cell are $D$ $=19.0 \mathrm{~cm}$ and $L=19.3 \mathrm{~cm}$, respectively. The sidewall is a 
Plexiglas tube with thickness $5 \mathrm{~mm}$. The corresponding aspect ratio $\Gamma$ (diameter/height) of the cell is thus unity. A square-shaped jacket made of flat glass plate is fitted to the outside of the sidewall [25]. The jacket is also filled with water, which greatly reduces the distortion effect to the PIV images caused by the curvature of the cylindrical sidewall. The top and bottom plates of the cell are made of 1-cm-thick copper with gold-plated surfaces. A stainless steel cover is attached on the top of the upper plate. This cover together with the upper surface of the top copper plate serves as a cooling chamber, which removes heat from the top surface via a refrigerated circulator (Hakke N6 KT-50W). Cold water is pumped to the chamber through two inlets and flows out through two outlets on the top of the chamber. The temperature stability of the refrigerated circulator is $0.01{ }^{\circ} \mathrm{C}$. Two silicon rubber film heaters connected in series are sandwiched to the back of the bottom plate to provide constant and uniform heating. A dc power supply (Xantrex XFR 300-4) with $99.99 \%$ long-term stability is used to provide the heating power. Two thermistors are embedded beneath the fluid-contact surface of each conducting plate to record the temperature of the plate.

The top and bottom parts of the cell are wrapped with nitrile rubber sheets for thermal insulation. The entire convection cell together with the jacket is placed inside a thermostat box whose temperature matches the mean temperature of the bulk fluid in the convection cell. The thermostat box reduces heat leakage and maintains the constant mean temperature of the working fluid in the cell and jacket so that it is not subject to room temperature fluctuations.

\section{B. The PIV measurements}

The PIV system consists of a dual neodymium-doped yttrium aluminum garnet (Nd:YAG) laser operated at a power of $80 \mathrm{~mJ}$ per pulse, light-sheet optics, a cooled CCD camera with 12-bit dynamic range and $1280 \times 1024$ spatial resolution, a synchronizer, and control software, which contains a PIV data analysis package (TSI, Inc.). A laser light sheet of thickness $\simeq 2 \mathrm{~mm}$ is used to illuminate the seed particles in the plane of interest. In PIV measurements the optimal seed particle size for an accurate determination of the particle position is such that the image of the particle roughly occupies the area of one pixel in the CCD sensor [38]. In our case the field of view (or measuring area) is $19 \times 19.3 \mathrm{~cm}^{2}$, which, when projected on the $\mathrm{CCD}$ surface, corresponds to $186 \times 186 \mu \mathrm{m}^{2}$ per pixel. The seed particles used in the experiment are $50-\mu \mathrm{m}$-diameter polyamid spheres (density $1.03 \mathrm{~g} / \mathrm{cm}^{3}$ ), which are adequate for the present study, and the measured velocities are in excellent agreement with those obtained from the LDV method (see Sec. III). A measure of how well the seed particles can follow the flow is the socalled relaxation time [38], which is the time to bring a particle at rest to a velocity equal to that of the fluid. For the particles used in the experiment the relaxation time is $7 \mu \mathrm{s}$, which is much shorter than the typical time scale $(\sim 10 \mathrm{~Hz})$ of velocity fluctuations in turbulent convection in the range of $\mathrm{Ra}$ and Pr studied here. A two-dimensional velocity map is obtained by cross-correlating two consecutive images taken
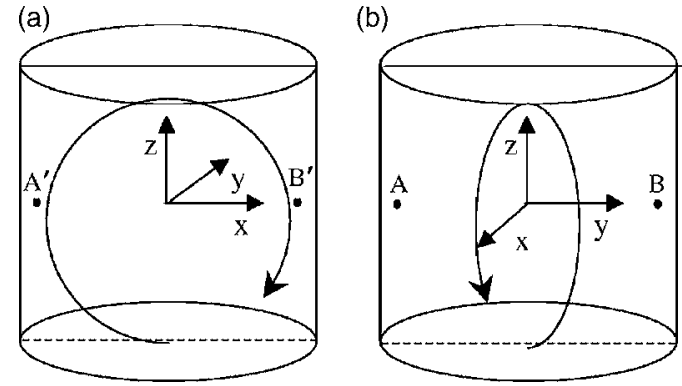

FIG. 1. Sketch of the convection cell and the Cartesian coordinates used in the velocity measurements: the view (a) facing and (b) perpendicular to the LSC plane. See text for the labels $A$ and $B$ and $A^{\prime}$ and $B^{\prime}$.

at a time interval that varies from 48 to $64 \mathrm{~ms}$ according to the value of $\mathrm{Ra}$ and is optimal for the corresponding flow speed. The selection criterion for the delay time is set such that the particles move several pixels across during the time interval. Each $2 \mathrm{D}$ velocity vector is calculated from a subwindow $(32 \times 32$ pixels) that has $50 \%$ overlap with its neighboring subwindows [36], so each vector corresponds to a region of $16 \times 16$ pixels on the surface of the CCD sensor. Because the field of view is of a square shape, the effective usable number of pixels is about $1024 \times 1024$, which would give $64 \times 64$ velocity vectors for a given measuring area. Due to edge effects and the fact that the measuring area is not a perfect square, the actual $2 \mathrm{D}$ vector map comprises $62 \times 63$ velocity vectors. This corresponds to a spatial resolution of $3.06 \mathrm{~mm}$. Typically, we acquire 15000 vector maps with a sampling rate of $2.2 \mathrm{~Hz}$ in a single measurement for each plane (duration $113 \mathrm{~min}$ ).

\section{Experimental conditions and parameters}

Figure 1 shows the coordinates of the velocity measurements with respect to the cell geometry: (a) the view of the LCS plane and (b) the view perpendicular to the LSC plane. The origin of the coordinates is chosen to coincide with the cell center. The $x$ and $z$ axes lie in the rotation plane of the LSC (the LSC plane) and the $y$ axis is perpendicular to that plane. The labels $A$ and $B$ and $A^{\prime}$ and $B^{\prime}$ will be explained in Sec. III. Ciliberto et al. have shown in an aspect-ratio-1 cell that the Nusselt number Nu is not affected appreciably when the cell is tilted by a small angle [39]. (Note, however, that Chillà et al. [40] and Sun et al. [41] have recently found that tilting the cell does affect the measured $\mathrm{Nu}$ in an aspectratio- 0.5 cell.) On the other hand, the effect of tilting to the velocity field in an aspect-ratio-1 cell has not been investigated systematically. To check the cell tilt effect, we first leveled the convection cell to be as horizontal as possible (better than $0.1^{\circ}$ ), and then measured the $2 \mathrm{D}$ velocity map in two vertical planes passing through the $z$ axis and perpendicular to each other. It is found that the velocity maps in the two planes are very different, indicating that the large-scale mean flow has a quasi-2D structure and its rotation plane has a preferred orientation. In this study we want to measure the properties of the velocity field in different planes with respect to the LSC, and therefore we tilt the cell by a small 
angle $\left(<1^{\circ}\right)$ in order to lock the LSC in the given $x z$ plane. This will prevent azimuthal drifts and occasional reversals of the LSC and thus eliminate ambiguities in the interpretation of the PIV results. Under this condition, we carry out the PIV measurements in the vertical planes at angles $0^{\circ}, 45^{\circ}$, and $90^{\circ}$ with respect to the rotation plane of the LSC (the $x z$ plane). The experiment was conducted at $\operatorname{Pr}=4.3$, which corresponds to a mean bulk fluid temperature of $40.0^{\circ} \mathrm{C}$. At this temperature the fluid material properties are $\alpha=3.85$ $\times 10^{-4} \mathrm{~K}^{-1}, \nu=6.58 \times 10^{-7} \mathrm{~m}^{2} / \mathrm{s}$, and $\kappa=1.52 \times 10^{-7} \mathrm{~m}^{2} / \mathrm{s}$. Two identical measurements were made for $\mathrm{Ra}=3.8 \times 10^{9}$ and $7.0 \times 10^{9}$, respectively, and the obtained results are qualitatively the same. Thus, only those for $\mathrm{Ra}=7.0 \times 10^{9}$ are presented below. At this $\mathrm{Ra}$, the temperature difference $\Delta T$ across the cell is $25.9^{\circ} \mathrm{C}$. Previous studies have shown that departures from the Boussinesq approximation for this value of $\Delta T$ are negligibly small [42]. For the present value of $\mathrm{Ra}$ the measured Nusselt number $\mathrm{Nu}=114.22$, which is in excellent agreement with the early result obtained also in a $\Gamma=1$ cylindrical cell at the same Prandtl number [43]. For the same cell and at the center of the bottom plate, both the thermal boundary layer thickness $\left(\delta_{t h}\right)$ and velocity boundary layer thickness $\left(\delta_{v}\right)$ have been previously measured at $\operatorname{Pr}$ $\approx 7$. For $\mathrm{Ra}=7.0 \times 10^{9}$, one finds $\delta_{t h}=0.67 \mathrm{~mm}$ [44] and $\delta_{v, \text { bottom }}=2.6 \mathrm{~mm}[5]$. For the velocity boundary layer near the sidewall, to our knowledge no experimental measurement has been made in the cylindrical geometry. However, Qiu and Xia [8] have previously measured the sidewall boundary layer in a cubic cell at $\operatorname{Pr}=7$ and the result from that measurement gives $\delta_{v \text {, sidewall }}=2.5 \mathrm{~mm}$ for $\mathrm{Ra}=7.0 \times 10^{9}$. This may be used as a rough estimate for the cylindrical cell. It is seen that all these length scales are smaller than the spatial resolution $(\simeq 3 \mathrm{~mm})$ of the present PIV measurement. As a result, the present experiment does not resolve features related to either the thermal or velocity boundary layers. Below, $u, v$, and $w$ are used to denote the instantaneous local velocity components along the $x, y$, and $z$ directions, respectively; and $U, V$, and $W$ are the corresponding time-averaged quantities.

\section{RESULTS AND DISCUSSION}

\section{A. Three-dimensional structure of the mean velocity field}

Figure 2 shows the time-averaged velocity map measured in the plane of the LSC (the $x z$ plane). It is seen that the overall flow pattern in the plane of the LSC is a tilted ellipse with its long axis pointing approximately to the cell diagonal. There are two very small vortices at the two opposite corners along the short axis of the ellipse. The outer region of the ellipse has a larger velocity magnitude than that in the central core region. The largest velocity is found in the two horizontal sidewall regions, which are located approximately (but not exactly) at the mid-height of the cell. When combined with the recent temperature [31,32] and flow visualization [25] measurements, we find that the outer region of the ellipse is the region in which most thermal plumes are concentrated. With the flow field shown in Fig. 2, one can trace the average motion of a typical thermal plume by following

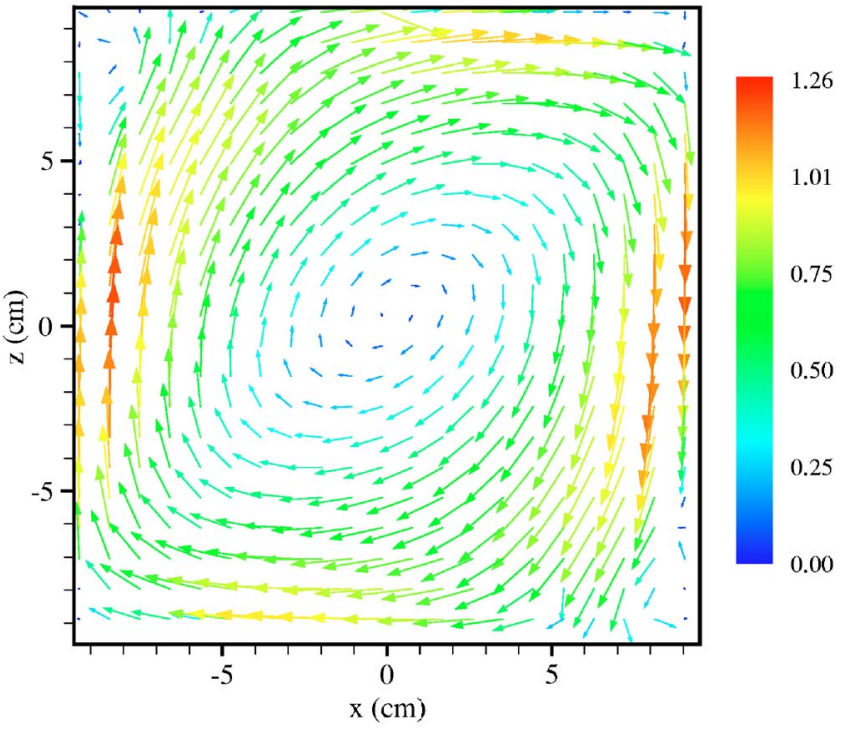

FIG. 2. (Color online) Time-averaged velocity vector map measured in the plane of the LSC at $\mathrm{Ra}=7.0 \times 10^{9}$. For clarity, a coarsegrained vector map of size $21 \times 21$ is shown. The magnitude of the velocity $\left(U^{2}+W^{2}\right)^{1 / 2}$ is coded in both gray scale (color) and the length of the arrows in units of $\mathrm{cm} / \mathrm{s}$. The time average is taken over a period of $113 \mathrm{~min}$ corresponding to 15000 velocity frames.

one of the elliptical orbits in the outer region. For example, when a warm plume starts to rise at the lower left corner of the cell, it is accelerated first by its own buoyancy and reaches its maximum speed in the left sidewall region at the mid-height of the cell. After reaching its maximum speed, the warm plume loses its steam because of thermal diffusion and turbulent mixing and thus is decelerated in the upper half region of the cell. Because of the continuity of the fluid flow, the velocity field in the upper half region of the cell has a large horizontal component, which sweeps the warm plume toward the middle of the cell. When the warm plume finally reaches the left side of the upper conducting plate, heat exchange takes place and the warm plume finishes its life cycle. In this way, the warm plume drives the flow mainly in the first half of its lifetime and is driven by the mean flow mostly in the last half of its life cycle. Similarly, a cold plume starts to fall at the upper right corner of the cell and goes through the same process along the other half of the elliptical orbit. Figure 2 thus provides the most direct and quantitative evidence to demonstrate the flywheel flow structure in the $\Gamma=1$ cylindrical cell, which was first proposed by Kadanoff et al. [22,23] and later modified by Qiu et al. $[32,33]$. A similar flow structure was also found in a rectangular shaped convection box [37].

To make a quantitative comparison with the previous LDV measurements [14], we plot the measured vertical velocity profile along the $x$ axis in Fig. 3(a) and the horizontal velocity profile along the $z$ axis in Fig. 3(b). The PIV results are obtained from the linear cut of the time-averaged 2D velocity map shown in Fig. 2. The LDV results were obtained in a unity-aspect-ratio cylinder of approximately the same dimensions as ours but with a slightly different Rayleigh number $\left(\mathrm{Ra}=3.7 \times 10^{9}\right)$ and Prandtl number $(\operatorname{Pr} \sim 5.4)$. As a result, the magnitudes of the velocity between the two 

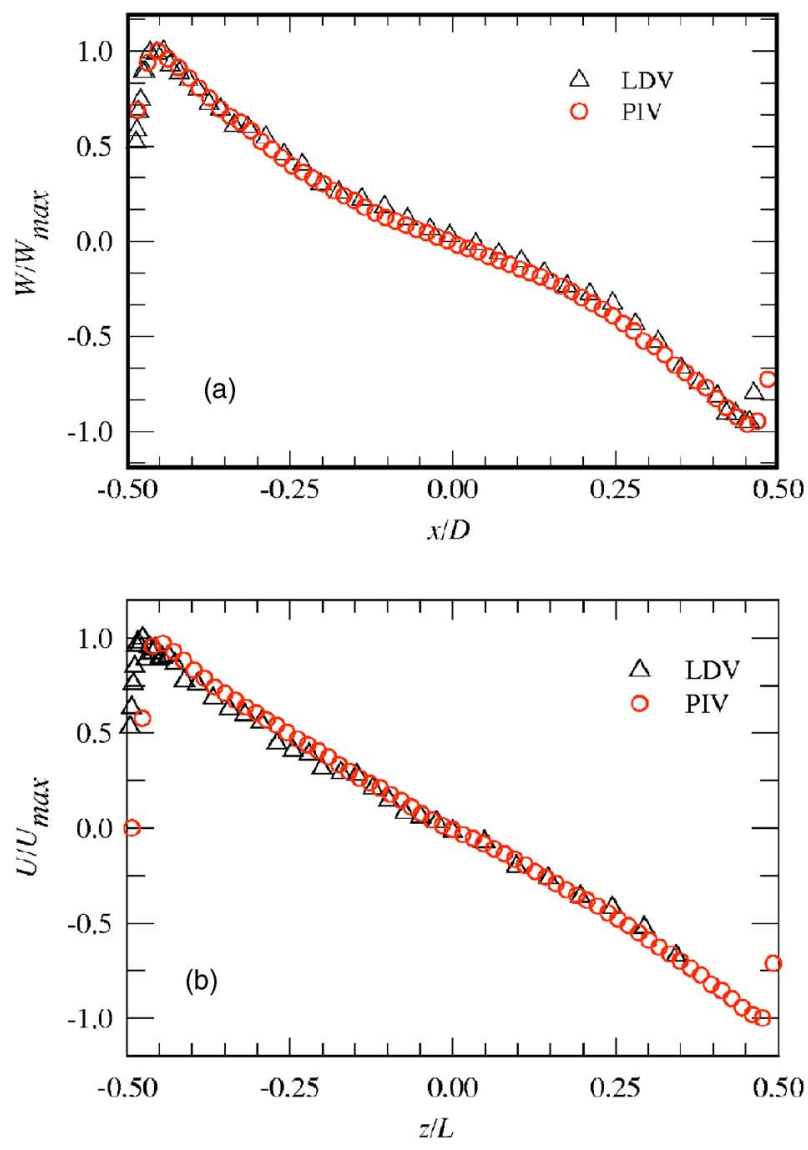

FIG. 3. (Color online) Comparison of the time-averaged velocity profiles measured with LDV (triangles) and PIV (circles). (a) Vertical velocity profile along the $x$ axis. (b) Horizontal velocity profile along the $z$ axis.

experiments are slightly different. In Fig. 3 we show the normalized velocity profiles with the velocity amplitude normalized by its maximum value and the length scales normalized by the cell dimensions. It is clearly seen that the velocity profiles measured with the two different techniques agree very well.

We now examine the flow field in the plane perpendicular to the LSC plane. Figure 4 shows the time series data of the vertical velocity fluctuations at position $A$ with $y=-7.7 \mathrm{~cm}$, $z=x=0$ [Fig. 4(a)] and at position $B$ with $y=7.7 \mathrm{~cm}, z=x$ $=0$ [Fig. 4(b)]. These two positions are located in the two opposing sidewall regions (see Fig. 1); both are in the plane perpendicular to LSC plane. It is seen that the vertical velocity in the sidewall region remains in one direction only for a short period of time $(\sim 15 \mathrm{~min})$. This reversal of flow direction is random over a longer period of time. This is contrary to the situation in the plane of the LSC, in which the vertical velocity in the sidewall region is locked in one direction most of the time. Careful examination of the time series data reveals that the reversals of the flow direction at the two opposite positions take place at approximately the same time, suggesting that the velocity field in the plane perpendicular to that of the LSC is still correlated in space. Similar behavior was also found for the temperature fluctuations in the region [32].
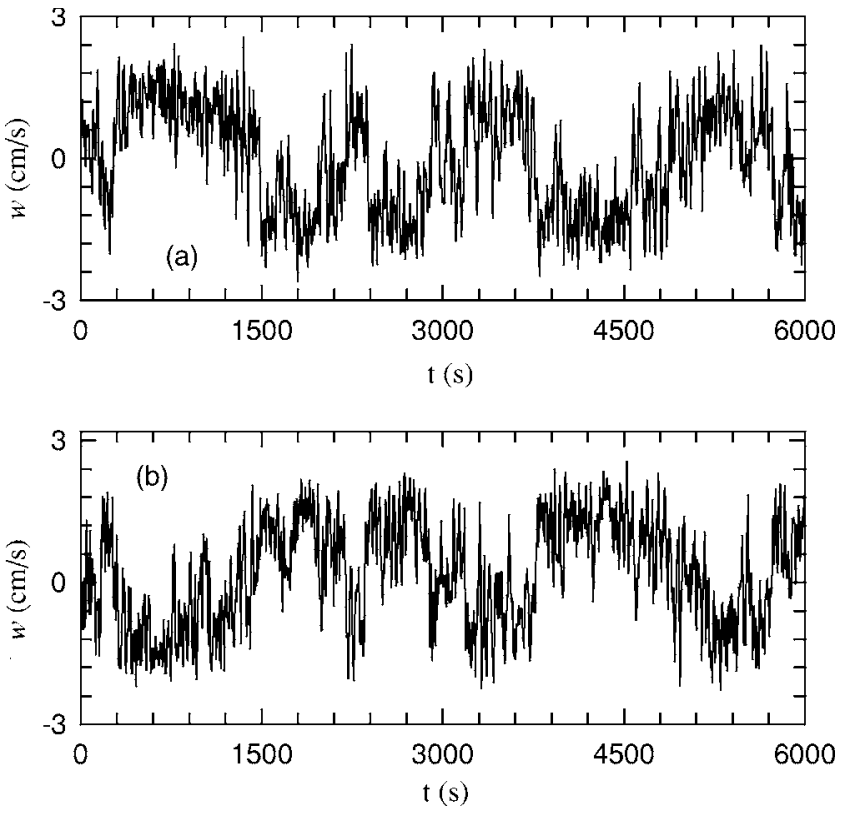

FIG. 4. Time series measurements of the vertical velocity fluctuations in the plane perpendicular to the LSC plane. The velocity measurements are taken at two opposing sidewall positions at the mid-height of the cell. Vertical velocity fluctuations at (a) position $A$ with $y=-7.7 \mathrm{~cm}, z=x=0$ and (b) position $B$ with $y=7.7 \mathrm{~cm}, z=x$ $=0$.

Figure 5(a) shows the velocity vector map time averaged only over a short period of time $(10 \mathrm{~min})$, during which the large-scale motion remains in one direction. It is seen that the flow pattern shown in Fig. 5(a) is very similar to that in the plane of the LSC, as shown in Fig. 2. Figure 5(b) shows the velocity vector map time averaged over another period of time $(10 \mathrm{~min})$, during which the large-scale motion has changed to an opposite direction. Except for the change of the rotational direction, the flow pattern shown in Fig. 5(b) is very similar to that shown in Fig. 5(a). Figures 5(a) and 5(b) thus reveal that the velocity field in the plane perpendicular to the LSC has two counter-rotating modes. The vector sum of the two flow patterns is shown in Fig. 5(c), which is essentially the same as the velocity vector map time averaged over a much longer period of time $(113 \mathrm{~min})$, as shown in Fig. 5(d). Because of the time-average effect, the final flow pattern shown in Fig. 5(d) is much different from that shown in Fig. 2. Figures 5(c) and 5(d) thus demonstrate that the reversal of the flow direction is indeed random, so that the velocity field in the plane perpendicular to the LSC plane has an equal probability to be in any one of the two counterrotating states. From the above PIV measurements together with the early temperature measurements [32], we conclude that the random reversal of the large-scale circulation in the plane perpendicular to the LSC plane is caused by the random positional switching of warm and cold plumes between the two opposing sides of the cell. Another feature that might be noted in Fig. 5(d) is that the rotational plane of the LSC is tilted slightly from vertical. This can be seen from fact that the time-averaged velocity vectors near the top and bottom plates in the central axis region $(y=0)$ are tilted slightly (toward the left near the top and right near the bottom). Xia, 
(a)

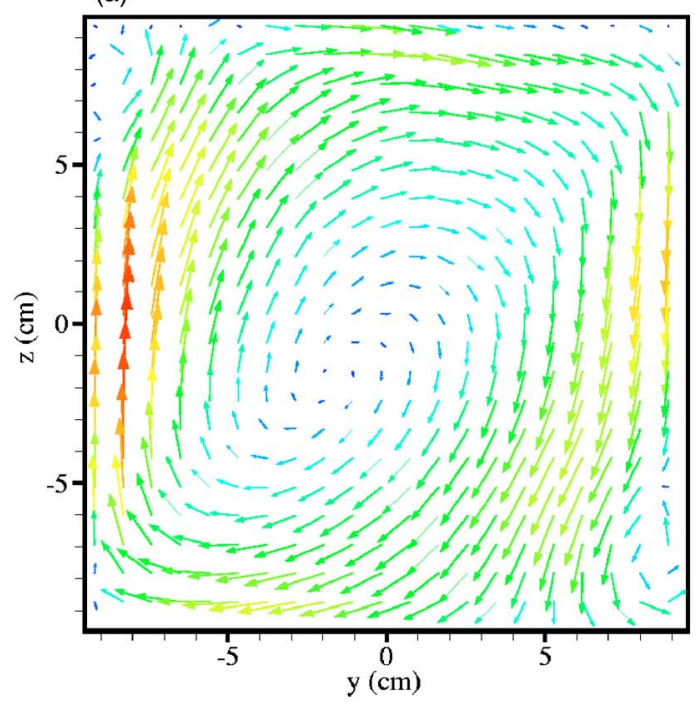

(c)

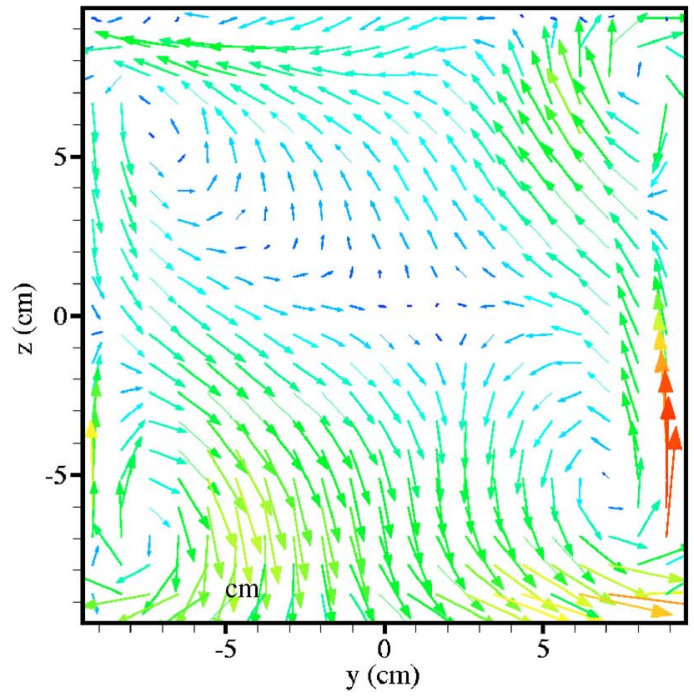

(b)

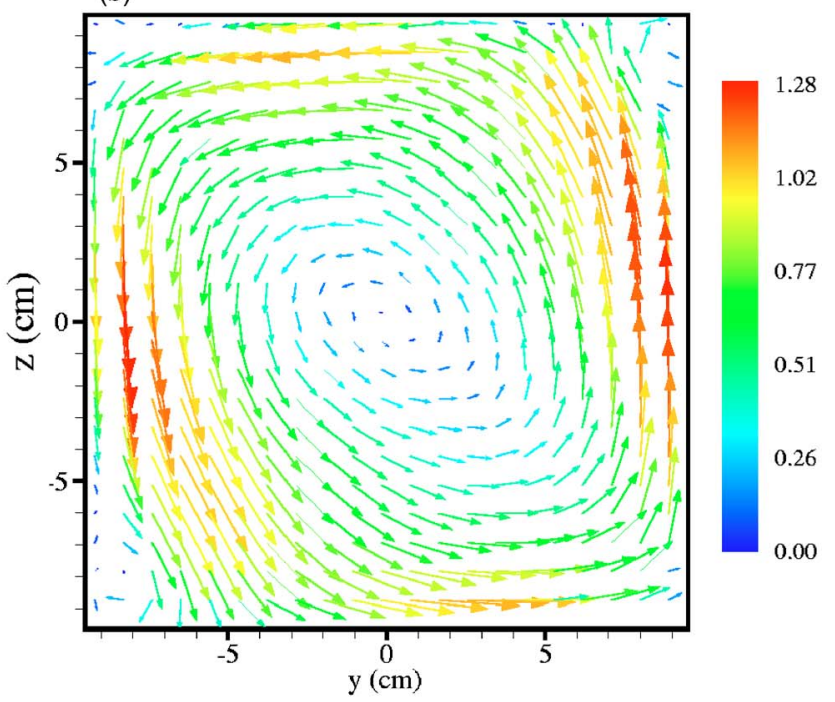

(d)

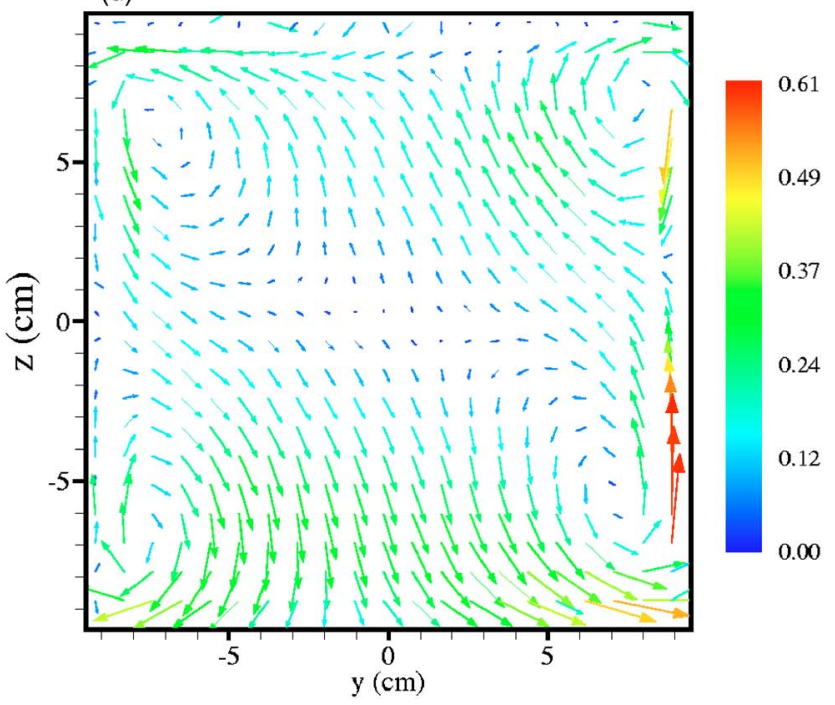

FIG. 5. (Color online) Short- and long-time-averaged velocity vector maps measured in the plane perpendicular to the LSC plane at $\mathrm{Ra}=7.0 \times 10^{9}$. For clarity, coarse-grained vector maps of size $21 \times 21$ are shown. The magnitude of the velocity $\left(V^{2}+W^{2}\right)^{1 / 2}$ is coded in both gray scale (color) and the length of the arrows in units of $\mathrm{cm} / \mathrm{s}$. (a) Time average is taken over a 10-min period during which the large-scale circulation remains (a) clockwise and (b) counterclockwise. (c) Vector average of the velocity vector maps shown in (a) and (b). (d) Long-time average over a period of 113 min corresponding to 15000 velocity frames.

Sun, and Zhou have previously found in a rectangular cell that the orientation of the LSC plane changed from being vertical to being diagonal when $\mathrm{Ra}$ became larger than $\sim 1 \times 10^{10}$ [37]. This tilting of the rotational plane of the mean wind at higher values of Ra suggests that the flow likes to be in a configuration having the largest spatial extent allowed by the geometry of the convection cell, as it corresponds to the most stable mode. It remains to be seen whether the same thing will happen in the cylindrical cell when Ra becomes sufficiently large.

Having understood the mean velocity field in the plane of the LSC and in the plane perpendicular to it, one might expect that the mean velocity field in other vertical planes passing through the $z$ axis should behave in a way somewhere intermediate between the two situations. Figure 6 shows the measured time-averaged velocity vector map in the plane at $45^{\circ}$ with respect to the LSC. By comparing Fig. 6 with Fig. 2 , we find that the flow pattern in the $45^{\circ}$ plane looks more like that in the $x z$ plane than that in the $y z$ plane. We understand this behavior with the following reasoning. First, in a previous experiment [44], Lui and Xia studied the spatial variation of the thermal boundary thickness, from which they concluded that the extent of the LSC in the $y$ direction (the width of the LSC) is approximately a half of the cell diameter. Our PIV measurements in the horizontal planes, which will be discussed below, also confirm this conclusion. As a result, the $45^{\circ}$ plane traverses mostly the band region of the LSC. Second, as discussed above, the flow pattern in the LSC plane is locked whereas the large-scale motion in the $y z$ plane shows random reversals. Therefore, the flow pattern in the plane of the LSC survives the time average and makes more contributions to that in the $45^{\circ}$ plane. These two factors 


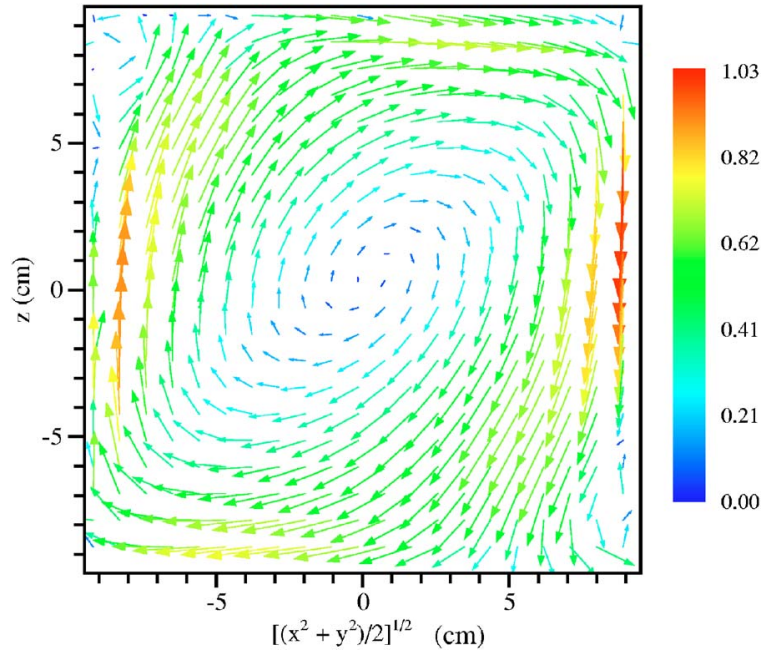

FIG. 6. (Color online) Time-averaged velocity vector map measured at $\mathrm{Ra}=7.0 \times 10^{9}$ in the plane at $45^{\circ}$ with respect to the LSC. For clarity, a coarse-grained vector map of size $21 \times 21$ is shown. The magnitude of the velocity is coded in both gray level (color) and the length of the arrows in units of $\mathrm{cm} / \mathrm{s}$. The time average is taken over a period of 113 min corresponding to 15000 velocity frames.

explain why the PIV measurement in the $45^{\circ}$ plane is essentially a projection of that in the LSC and the two look qualitatively the same. Still, there are some differences between Fig. 6 and Fig. 2. First, the maximum velocity in the $45^{\circ}$ plane $(\simeq 1.03 \mathrm{~cm} / \mathrm{s})$ is only about $80 \%$ of that in the plane of the $\operatorname{LSC}(\simeq 1.26 \mathrm{~cm} / \mathrm{s})$. Second, the two counter-rotating vortices at the corners of the cell are now larger than those in the LSC plane, reflecting the influence of the flow in the perpendicular plane. In addition, the size of the elliptical core region in the $45^{\circ}$ plane is larger than that in the plane of the LSC.

The three-dimensional structure of the flow field discussed above is a time-averaged result, which ignored the time variation of the flow field in the closed cell. While the long-time average is a powerful tool to filter out random noise, it may also average out some interesting dynamics in the flow field. To further study the dynamics in turbulent convection, one needs to examine the time dependence of the velocity field. In the following sections, we focus our discussion on the dynamics of the velocity field.

\section{B. Synchronized motion of thermal plumes between the top and bottom plates}

It has long been recognized that thermal plumes play an important role in determining the dynamics in turbulent convection. Zocchi et al. [24] identified three types of coherent structures in the convective flow: waves along the boundary layer, plumes, and spiraling swirls. They described a "life cycle" for these coherent structures in the closed cell. The recent experiments by Qiu et al. [32,33] confirmed the physical picture in Villermaux's model [30] that the thermal plumes are emitted alternately between the upper and lower conducting plates, producing a well-defined oscillation in
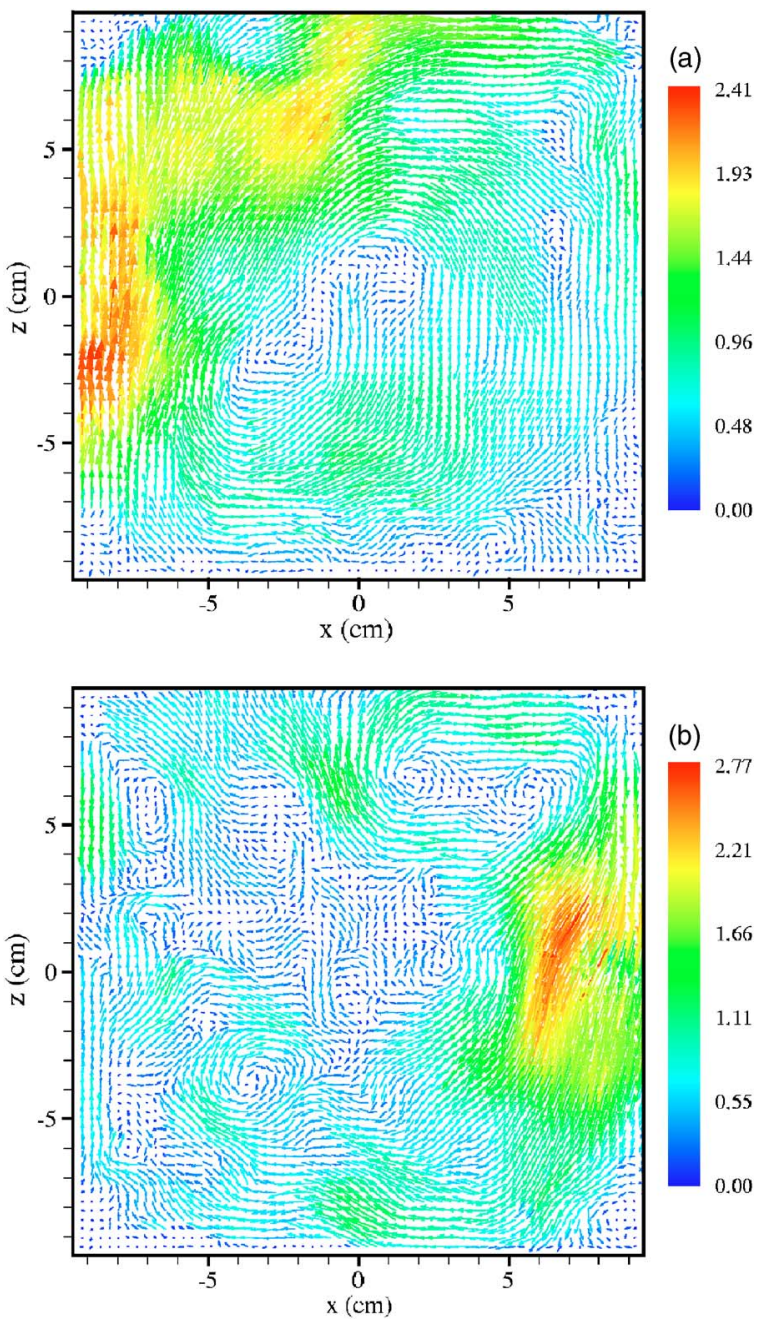

FIG. 7. (Color online) Instantaneous velocity vector maps taken in the plane of the LSC at $\mathrm{Ra}=7.0 \times 10^{9}$. The velocity maps are shown with the resolution $62 \times 63$. The magnitude of the velocity $\left(u^{2}+w^{2}\right)^{1 / 2}$ is coded in both gray level (color) and the length of the arrows in units of $\mathrm{cm} / \mathrm{s}$. Instantaneous velocity map taken at (a) an early time and (b) $20 \mathrm{~s}$ later.

both temperature and velocity signals. While we do not have an accurate imaging technique at the moment to visualize the bursting events of the thermal plumes in a $2 \mathrm{D}$ temperature field, the PIV technique provides us an alternative way to watch the consequence of the plume bursting in a $2 \mathrm{D}$ velocity field.

Figure 7 shows two examples of the instantaneous velocity vector map measured in the plane of the LSC. The time delay between the two images shown in Fig. 7(a) and Fig. 7 (b) is $20 \mathrm{~s}$, which is approximately one-half of the oscillation period $\left(\tau_{0} \sim 42 \mathrm{~s}\right)$ at this Ra. It is seen from Fig. $7(\mathrm{a})$ that there is a large velocity burst moving upward near the left sidewall. From the size of the velocity burst, we find that it is associated with a large cluster of warm plumes in the region and the magnitude of the velocity $(w \simeq 2.4 \mathrm{~cm} / \mathrm{s})$ is much larger than the relatively quiet background at the moment. The situation is reversed in Fig. 7(b), in which one sees a large velocity burst moving downward near the right sidewall. This velocity burst is associated with a cluster of 


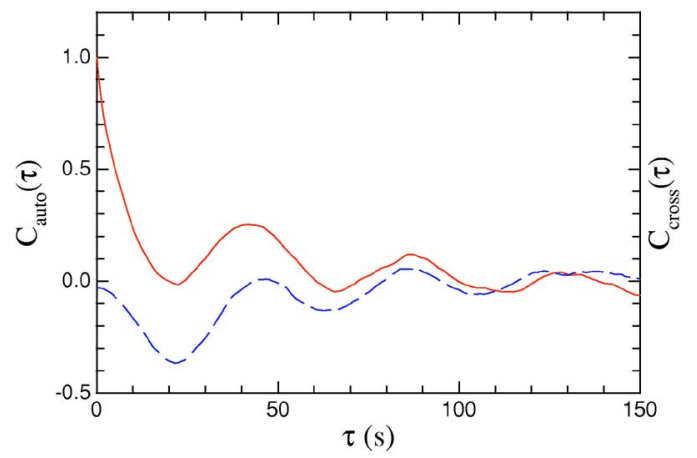

FIG. 8. (Color online) Measured autocorrelation function $C_{\text {auto }}(\tau)$ at position $A^{\prime}$ with $x=-7.7 \mathrm{~cm}, z=y=0$ (solid curve) and the cross-correlation function $C_{\text {cross }}(\tau)$ between positions $A^{\prime}$ and $B^{\prime}$ with $x=7.7 \mathrm{~cm}, z=y=0$ (dashed curve) as a function of delay time $\tau$ at $\mathrm{Ra}=7.0 \times 10^{9}$.

cold plumes in the region and the velocity field in other regions of the cell is rather quiet. By watching the movies of the velocity field generated by continuous PIV imaging [45], we find that this velocity bursting occurs alternately between the opposing sidewall regions. The upward going and downward going velocity bursts rarely appear at the same time; rather they almost always have a time delay of $\sim 20 \mathrm{~s}$.

To measure the delay time more accurately, we select two positions $A^{\prime}(x=-7.7 \mathrm{~cm}, z=y=0)$ and $B^{\prime}(x=7.7 \mathrm{~cm}, z=y$ $=0$ ) (see Fig. 1) in the plane of the LSC and calculate the auto- and cross-correlation functions of the vertical velocity $w$ between the two positions, which are located in the two opposite sidewall regions (both $2 \mathrm{~cm}$ from the wall and at the mid-height of the cell). Figure 8 shows the velocity autocorrelation function $C_{\text {auto }}(\tau)=\left\langle w_{A^{\prime}}(t+\tau) w_{A^{\prime}}(t)\right\rangle / \sigma\left(w_{A^{\prime}}\right)^{2}$ at position $A^{\prime}$ (solid curve) and the cross-correlation function $C_{\text {cross }}(\tau)=\left\langle w_{A^{\prime}}(t+\tau) w_{B^{\prime}}(t)\right\rangle /\left(\sigma\left(w_{A^{\prime}}\right) \sigma\left(w_{B^{\prime}}\right)\right)$ between positions $A^{\prime}$ and $B^{\prime}$ (dashed curve). Here $\sigma\left(w_{A^{\prime}}\right)\left[\sigma\left(w_{B^{\prime}}\right)\right]$ is the rms value of the vertical velocity $w_{A^{\prime}}\left[w_{B^{\prime}}\right]$ at the position $A^{\prime}$ $\left[B^{\prime}\right]$. Both $C_{\text {auto }}(\tau)$ and $C_{\text {cross }}(\tau)$ show a well-defined oscillation with the same oscillation period $\tau_{0}=42 \mathrm{~s}$. It is seen that the vertical velocity remains correlated for many periods, indicating that the periodic oscillation in the flow field is highly coherent. The measured $C_{\text {cross }}(\tau)$ is negative for small values of $\tau$ and reaches its minimum value at a delay time $\simeq \tau_{0} / 2$. This is because the velocity bursts at positions $A$ and $B$ have an opposite sign and they appear in these two positions with a delay $\tau_{0} / 2$. Figure 8 thus provides a strong support to the physical picture discussed above that synchronized alternating emission of thermal plumes takes place between the top and bottom conducting plates. Similar behavior was also found for the temperature auto- and crosscorrelation functions [31]. The fact that both the temperature and velocity correlation functions have the same behavior suggests that the velocity bursts shown in Fig. 7 are indeed generated by the thermal plumes.

An important prediction of Villermaux's model [30] is that the oscillation period $\tau_{0}$ is twice the cell crossing time $t_{0}=L /\langle w\rangle$, where $\langle w\rangle$ is the spatial-averaged rising (or falling) speed of the thermal plumes. In a recent experiment $[31,32]$, Qiu and Tong showed that the oscillation period $\tau_{0}$

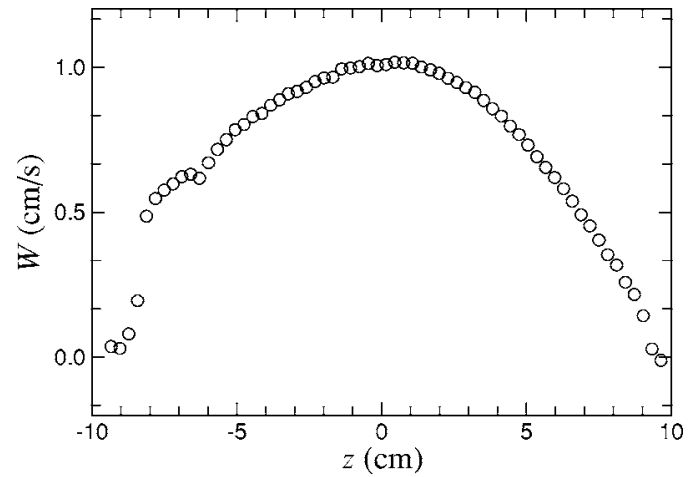

FIG. 9. Time-averaged vertical velocity profile $W(z)$ as a function of $z$ at $\mathrm{Ra}=7.0 \times 10^{9}$. The velocity profile is taken in the plane of the LSC with $x=-7.7 \mathrm{~cm}, y=0$.

is indeed very close to $2 t_{0}$, clarifying a previous misconception that the oscillation period equals to the cell circulation time $4 t_{0}$. In their experiment, however, the time-averaged vertical velocity at mid-height of the cell is used as $\langle w\rangle$ to estimate the cell-crossing time $t_{0}$. As shown in Fig. 2, the plume's velocity in fact varies as it traverses the height of the cell. With the $2 \mathrm{D}$ velocity map, we can now make more accurate estimate of the cell-crossing time $t_{0}$. Figure 9 shows a vertical scan of the vertical velocity profile $W(z)$ taken at $2 \mathrm{~cm}$ from the sidewall. It is seen that the plume's vertical motion is characterized by an initial acceleration by its own buoyancy, when the plume leaves the lower conducting plate, reaching a maximum velocity at the mid-height of the cell, and then decelerating, due to thermal diffusion and turbulent mixing, when the plume approaches the upper conducting plate. Integrating the inverse of this velocity profile from the bottom to the top plate gives us a good estimation of the cell-crossing time, i.e., $t_{0}=\int_{0}^{L}[1 / W(z)] d z=22 \mathrm{~s}$, which is in excellent agreement with the one-half of the oscillation period $\tau_{0}$ obtained above.

An important issue that was not discussed in Villermaux's model is how the arriving warm (or cold) plumes on one side of the conducting plate trigger the emission of cold (or warm) plumes on the other side of the same plate. Our PIV measurements and the early temperature measurements $[31,32]$ show that the triggering takes place in a very short time period, almost negligible when compared with the cellcrossing time $t_{0}$. Therefore, the oscillation period is just twice the cell-crossing time. A possible triggering mechanism is that when the incoming plumes impinge on the conducting surface, they generate a pressure wave at the thermal boundary layer and the wave propagates to the other side of the cell to trigger the plume emission there. In fact, such waves "propagating along boundary layers" have been observed by Zocchi et al. [24] and more recently by Haramina and Tilgner [35]. Further experimental studies are needed to reveal the detailed dynamics near the thermal boundary layers.

It should be emphasized that the triggering event almost always leads to the bursting of a cluster of plumes, not just a single one. We can thus classify plumes into two categories according to their properties of spatial organization. The first is clustered ones that are generated by the synchronized 
plume emissions and always travel along the opposite sidewalls. The motions of this type are thus spatially organized. The second category is individual plumes that are generated randomly at the conducting plates and they travel across the cell without spatial organization. Evidence for the clustering of plumes has been found experimentally from previous flow visualization, shadowgraph and PIV measurements $[14,25,37]$ and also from measurements of the local heat flux $[46,47]$. More recently, Parodi et al. also found numerical evidence for plume clustering [48]. The clustered plumes, whether they physically merge together to form larger (or composite) plumes or simply stay in close proximity by traveling as a pack will suffer less heat loss and are better able to survive the shearing and mixing of the turbulent flow. In contrast, the unorganized plumes have shorter lifetime due to thermal diffusion and shear or mixing by the turbulent flow and thus have less chance to reach the opposite plate. This explains why it is the organized or clustered plumes that contribute predominantly in heat transfer, as found by local heat flux measurements $[46,47]$.

\section{Spatial coherence of bulk velocity oscillations}

An important question one might ask is how does the alternating emission of thermal plumes between the upper and lower conducting surfaces affect the overall fluid motion in the closed cell? Villermaux's model [30] did not discuss this issue. The recent experiment by Qiu et al. [33] showed that the thermal plumes interact with the convecting fluid in a closed cell in two different modes. First, they directly entrain the surrounding fluid in the sidewall region along the vertical direction. This is supported by the experimental finding that the vertical velocity oscillation occurs mainly in the plume-dominated sidewall region and its phase remains the same as that of the temperature oscillation. Second, the interaction between the thermal plumes and the bulk fluid in the central region takes place via hydrodynamic forces, which introduce a phase shift to the two horizontal velocity components in the region. It was found that the velocity oscillation in the central region of the $\Gamma=1$ cell is predominantly in the horizontal direction.

To further understand the coupling between the plumes' vertical motion and the horizontal velocity oscillations in the bulk region, one needs to carry out simultaneous multipoint velocity measurements. With the PIV technique, we are able to measure the $2 \mathrm{D}$ velocity field in two orthogonal planes $(x z$ and $y z$ ) and obtain the phase relationship between velocity components either in the same direction or in different directions at the same and different positions. The phase difference between two velocities $\mathbf{v}\left(\mathbf{r}_{1}\right)$ and $\mathbf{v}\left(\mathbf{r}_{2}\right)$, as measured by the cross-correlation function $C_{\text {cross }}(\tau)$ of the two velocities such as that shown in Fig. 8, is given by $\varphi\left(\mathbf{v}\left(\mathbf{r}_{1}\right), \mathbf{v}\left(\mathbf{r}_{2}\right)\right)$ $\equiv 2 \pi f_{0} \tau_{1}$ where $f_{0}=1 / \tau_{0}$ is the frequency of the bulk velocity oscillation and $\tau_{1}$ is the position of the first maximum of the absolute value of $C_{\text {cross }}(\tau)$. In the following, we present the measured profiles of $\varphi$ along the axes $x, y$, and $z$, with the measuring position of one of the velocities being fixed at the cell center and the position of the other velocity varying along the respective axes.
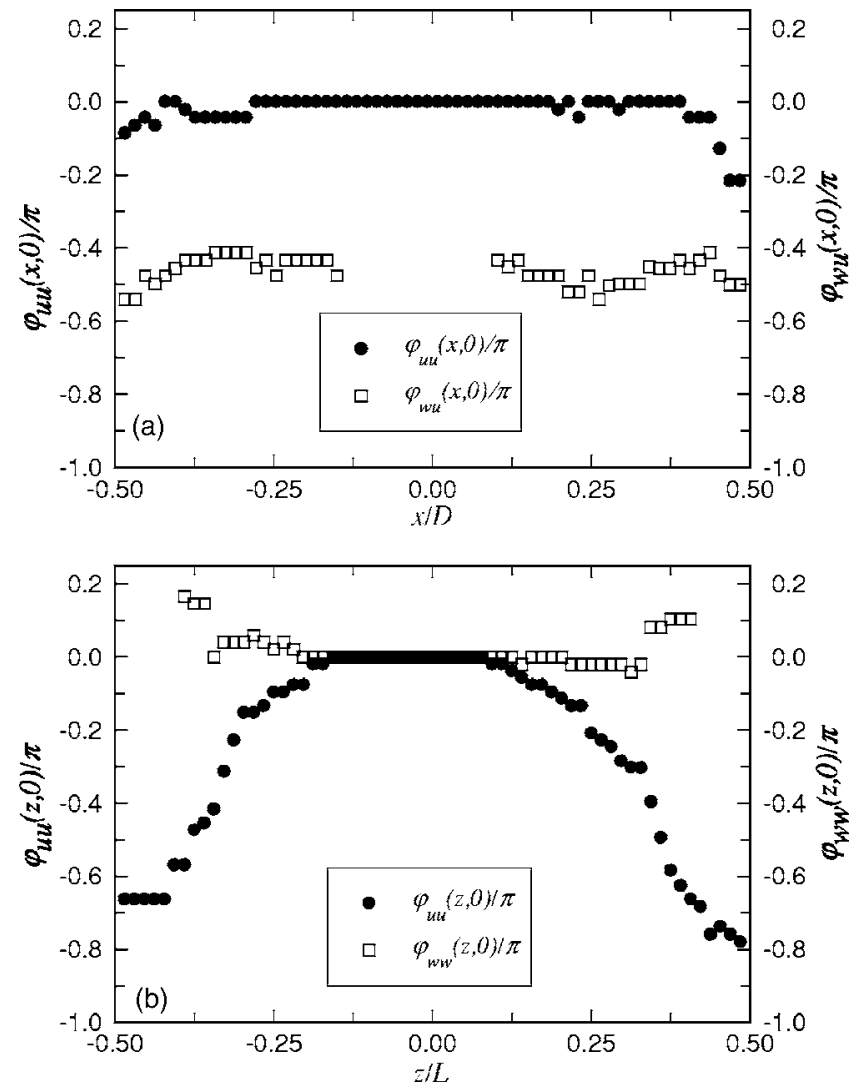

FIG. 10. Measured profiles of the phase difference in the LSC plane at $\mathrm{Ra}=7.0 \times 10^{9}$. (a) $\varphi_{u u}(x, 0)=\varphi(u(x), u(0))$ (closed circles) and $\varphi_{w u}(x, 0)=\varphi(w(x), u(0))$ (open squares) along the $x$ axis. (b) $\varphi_{u u}(z, 0)=\varphi(u(z), u(0)) \quad\left(\right.$ closed circles) and $\varphi_{w w}(z, 0)$ $=\varphi(w(z), w(0))$ (open squares) along the $z$ axis.

We first examine the situation in the LSC plane (the $x z$ plane). Figure 10(a) shows the measured $\varphi_{u u}(x, 0)$ $\equiv \varphi(u(x), u(0)) \quad$ (closed $\quad$ circles) $\quad$ and $\varphi_{w u}(x, 0)$ $\equiv \varphi(w(x), u(0))$ (open squares) along the $x$ axis. In the plot, $\varphi$ is normalized by $\pi$ and $x$ is normalized by the cell diameter $D$. The measured $\varphi_{u u}$ clearly shows that the in-plane horizontal velocity oscillations at the mid-height of the cell are highly correlated and they have a common phase across the entire cell diameter. The measured $\varphi_{w u}$ tells us that the horizontal motion at the cell center is a quarter of a period $(\pi / 2)$ ahead of the vertical motion near the sidewall. Because the vertical motion of the plumes is confined mainly in the sidewall region, its correlation with the resulting horizontal motion at the cell center decays as the measuring position of $w(x)$ is moved toward the cell center. In the central region of the cell, $C_{\text {cross }}(\tau)$ between $w(x)$ and $u(0)$ is so small that no reliable phase information can be obtained. As a result, no data point for $\varphi_{w u}$ is shown in the central region of the cell. A similar decay was also observed for the cross-correlation function between the temperature and vertical velocity fluctuations [33]. Closed circles in Fig. 10(b) show the measured $\varphi_{u u}(z, 0) \equiv \varphi(u(z), u(0))$ along the $z$ axis. It is seen that the horizontal velocity oscillations remain in phase along the $z$ axis mainly in the middle one-half of the cell. The horizontal velocity oscillations near the upper and lower conducting 


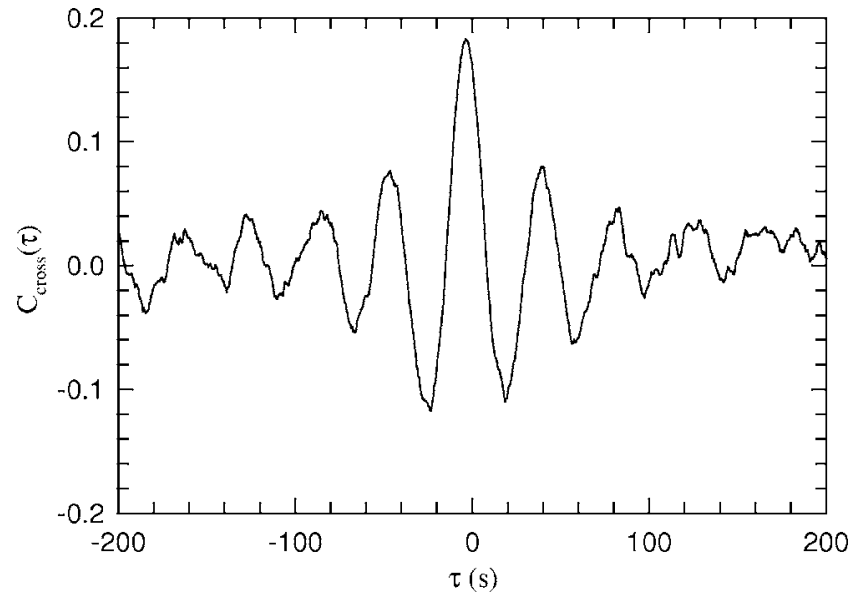

FIG. 11. Cross-correlation function $C_{w u}(\tau)$ between the two velocities $w(x=-8.5 \mathrm{~cm}, z=-8.5 \mathrm{~cm})$ and $u(x=0, z=0)$ as a function of delay time $\tau$. The measurement is made in the LSC plane at $\mathrm{Ra}=7.0 \times 10^{9}$.

plates gradually lag behind those in the central region of the cell. As shown in Fig. 9, the vertical velocity of the rising (or falling) plumes remains above $80 \%$ of their maximum value in the middle one-half of the cell and thus one may expect that the thermal plumes push the bulk fluid more vigorously in this region.

To better understand Fig. 10, let us consider a burst of warm plumes ascending from the lower left corner of the cell. As the plumes move vertically along the left sidewall, they push the bulk fluid to the right. As shown in Fig. 2, the plumes' vertical velocity reaches maximum at the mid-height of the cell after they traveled for a quarter of a period. At the same moment the horizontal velocity of the bulk fluid (to the right) also reaches its maximum, which explains why $w$ and $u$ have maximum correlation at the phase delay of $\pi / 2$. Indeed, one can see this interaction quantitatively by examining the phase relationship between $w$ at the lower left corner of the cell and $u$ at the cell center. Figure 11 shows the cross-correlation function $C_{w u}(\tau)$ between the two velocities $w(x=-8.5 \mathrm{~cm}, z=-8.5 \mathrm{~cm})$ and $u(x=0, z=0)$. It is seen clearly that $w$ and $u$ at the two positions have the same phase $\left[C_{w u}(\tau)\right.$ peaks at $\left.\tau \simeq 0\right]$. After another quarter of a period, the warm plumes reach the top plate and the rightward motion of the bulk fluid stops. At almost the same time, a burst of cold plumes is triggered at the upper right corner of the cell and they start to move downward along the right sidewall, pushing the bulk fluid to the left to complete the cycle. This explains why $\varphi_{w u}$ equals $\pi / 2$ for both the positive and negative values of $x$. Figures 10 and 11 thus demonstrate convincingly that the horizontal oscillation of the bulk fluid in the $x$ direction is driven by the synchronized vertical motion of the rising and falling plumes along the two opposing sidewall regions. The entire process is illustrated in the sketch shown in Fig. 12.

We now discuss the situation in the $y z$ plane, the plane perpendicular to the LSC plane. Figure 13(a) shows $\varphi_{v v}(y, 0) \equiv \varphi(v(y), v(0)) \quad$ (closed circles) and $\varphi_{w v}(y, 0)$ $\equiv \varphi(w(y), v(0))$ (open squares) along the $y$ axis. Figure 13(b) shows $\varphi_{v v}(z, 0) \equiv \varphi(v(z), v(0))$ along the $z$ axis. The mea-
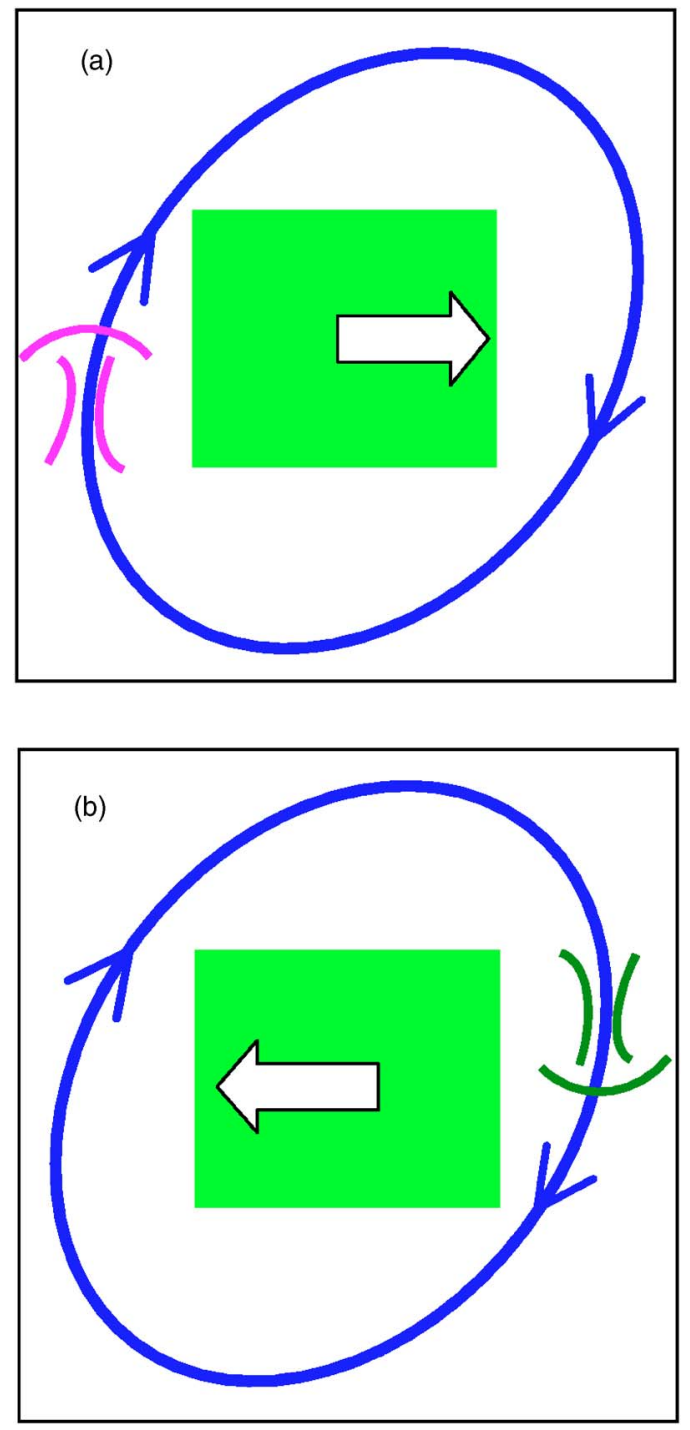

FIG. 12. (Color online) A sketch showing the interaction between the vertical motion of a burst of plumes and the horizontal motion of the bulk fluid in the LSC plane. (a) A burst of rising plumes on the left pushing the bulk fluid to the right. (b) A burst of falling plumes on the right pushing the bulk fluid to the left.

sured $\varphi_{v v}(y, 0), \varphi_{w v}(y, 0)$, and $\varphi_{v v}(z, 0)$ all show similar behavior to that of $\varphi_{u u}(x, 0), \varphi_{w u}(x, 0)$, and $\varphi_{u u}(z, 0)$ in the LSC plane, indicating that the spatial coherence and the driving mechanism of the horizontal bulk oscillation in the $y$ direction remain the same as those in the $x$ direction. It has been shown in Fig. 5 that the rising and falling plumes in the $y z$ plane produce a similar flow pattern to that in the LSC plane. The only difference is that the large-scale flow in the $y z$ plane is not locked and has two counter-rotating modes. Therefore, we expect that when the large-scale flow is in the clockwise rotation mode, as shown in Fig. 5(a), the interaction between the vertical motion of a burst of plumes and the horizontal motion of the bulk fluid remains the same as that shown in Fig. 12. When the large-scale flow is in the counterclockwise rotation mode, as shown in Fig. 5(b), the process is reversed as shown in Fig. 14. 

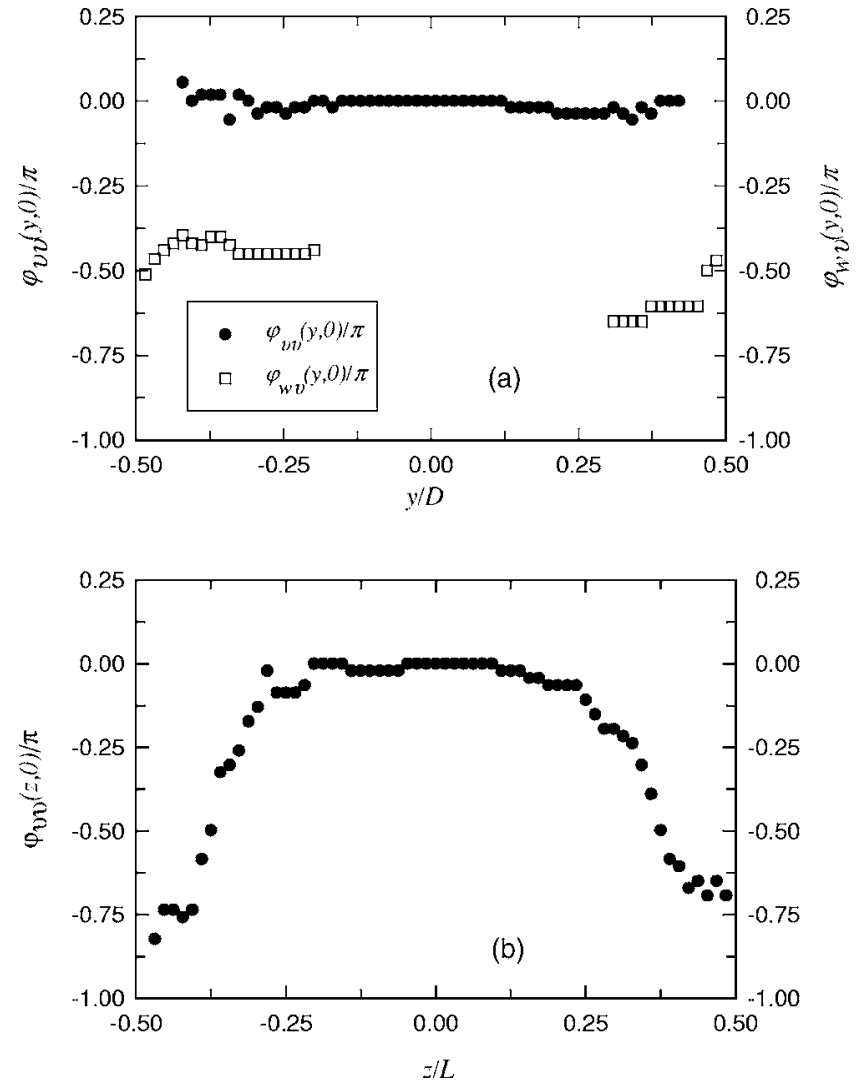

FIG. 13. Profiles of the phase difference in the plane perpendicular to the LSC plane at $\mathrm{Ra}=7.0 \times 10^{9}$. (a) $\varphi_{v v}(y, 0)$ $=\varphi(v(y), v(0))\left(\right.$ closed circles) and $\varphi_{w v}(y, 0)=\varphi(w(y), v(0))$ (open squares) along the y axis. (b) $\varphi_{v v}(z, 0)=\varphi(v(z), v(0))$ along the $z$ axis.

In addition to their pushing of the bulk fluid to oscillate horizontally, the rising and falling plumes along the sidewall also generate vertical motions via their shear. It has been found previously that this shear-driven motion weakens toward the cell center, making it only very weakly oscillatory in time in the central region $[28,29,33]$. From $\varphi_{w w}(x, 0)$ and $\varphi_{w w}(y, 0)$ shown in Fig. 15, it is seen that this small vertical motion remains spatially coherent in both the $x$ and $y$ directions for less than half of the cell dimension. Because it is driven by plumes moving along the sidewall from top to bottom, the vertical motion remains spatially coherent in the vertical direction over much of the height of the cell as shown by $\varphi_{w w}(z, 0)[$ Fig. $10(\mathrm{~b})]$. While the vertical motion in the central region is spatially coherent, it is not time periodic (or only very weakly). This is what sets it apart from motions in the two horizontal directions in the central region. This can be seen in Fig. 16(a), where we plot the autocorrelation function (dashed line) of the vertical velocity $w$ at cell center and the cross-correlation function (solid line) between vertical velocities at cell center and that at $z=4.2 \mathrm{~cm}$ on the central axis. The figure shows clearly that the vertical motions at the two positions on the central axis are in phase; however, the motion itself is not temporally oscillatory. In contrast, the motions in the two horizontal directions remain temporally coherent over many oscillating periods, which are shown in Fig. 16(b) by the autocorrelation functions of $u$ (solid line) and of $v$ (dashed line) at the cell center.
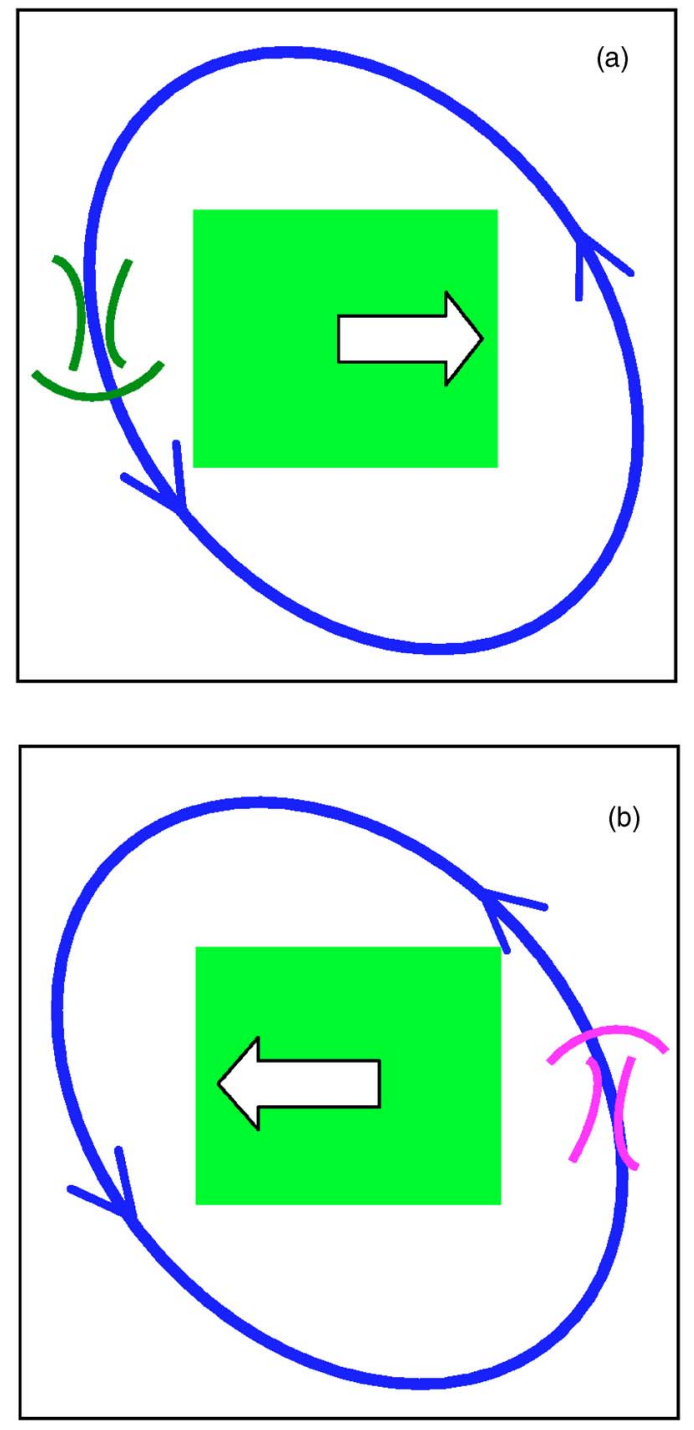

FIG. 14. (Color online) A sketch showing the interaction between the vertical motion of a burst of plumes and the horizontal motion of the bulk fluid in the $y z$ plane, which is perpendicular to the LSC plane. (a) A burst of falling plumes on the left pushing the bulk fluid to the right. (b) A burst of rising plumes on the right pushing the bulk fluid to the left.

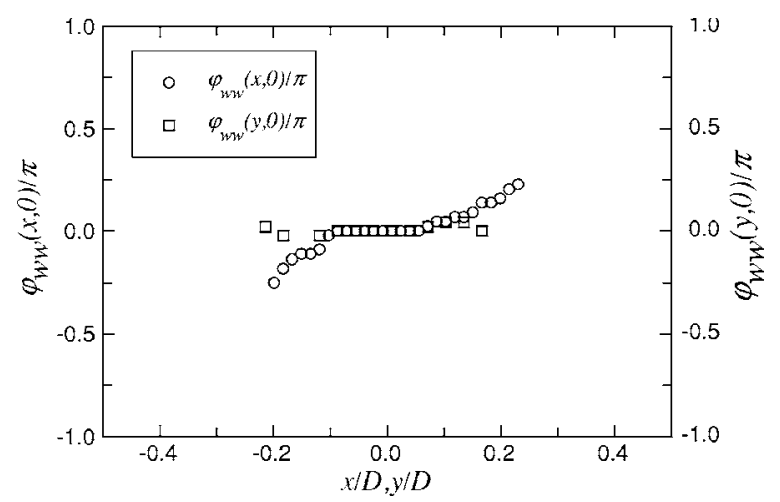

FIG. 15. Profiles of the phase difference of vertical velocity at $\mathrm{Ra}=7.0 \times 10^{9} . \varphi_{w w}(x, 0)=\varphi(w(x), w(0))$ (open circles) along the $x$ axis and $\varphi_{w w}(y, 0)=\varphi(w(y), w(0))$ (open squares) along the $y$ axis. 

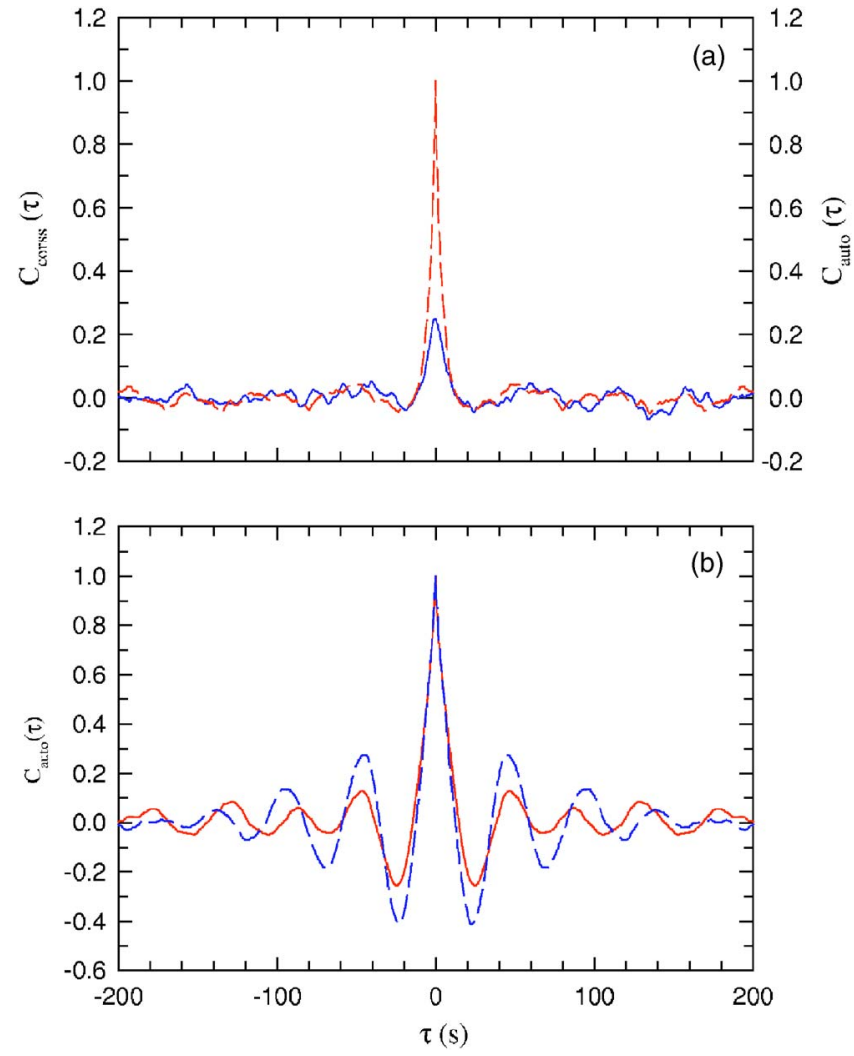

FIG. 16. (Color online) Autocorrelation function $C_{\text {auto }}(\tau)$ and cross-correlation function $C_{\text {cross }}(\tau)$ of velocity components. (a) $C_{\text {cross }}(\tau)$ between $w$ at $z=4.2 \mathrm{~cm}, x=y=0$ and $w$ at $z=x=y=0$ (solid line); $C_{\text {auto }}(\tau)$ of $w$ at $x=y=z=0$ (dashed line). (b) $C_{\text {auto }}(\tau)$ of $u$ at $x=y=z=0$ (solid line); $C_{\text {auto }}(\tau)$ of $v$ at $x=y=z=0$ (dashed line).

\section{CONCLUSION}

We have made a systematic investigation of the threedimensional flow structures and dynamics of turbulent Rayleigh-Bénard convection in an aspect-ratio-1 cylindrical cell using water as the convecting fluid. Instantaneous twodimensional velocity vector maps are measured using the technique of particle image velocimetry. The measurements are carried out at a fixed $\operatorname{Ra}\left(=7 \times 10^{9}\right)$ and $\operatorname{Pr}(=4.3)$ and in different vertical cross-sectional planes of the cell. The experiment clearly reveals how the thermal plumes are synchronized in their emissions and are organized spatially in their motions between the upper and lower conducting plates, leading to an oscillatory motion in the bulk region of the fluid with an oscillation period equal to twice of the plume's cell-crossing time. This result provides a direct verification of the theoretical model proposed by Villermaux [30].

From the measured instantaneous velocity vector map, we find the phase relationship between the velocity components along different directions and at different positions in a 2D plane. This allows us to study the spatial coherence of the bulk velocity oscillations. It is found that the fluid motion in the bulk region remains highly correlated over a large spatial extent. From the measured phase difference between the vertical velocity near the sidewall and the horizontal velocity at the cell center, we demonstrate how the rising and falling plumes generate horizontal velocity oscillations in the bulk region of the fluid. The experiment thus provides a fascinating example on how a highly organized and coherent flow motion can emerge from a closed turbulence system. The present study of the three-dimensional flow structures and dynamics of turbulent convection is focused on the bulk region. To further understand how the organized bulk fluid motion interacts with the conducting surface, one needs to carry out simultaneous PIV measurements of the flow field near the boundary layer in the planes both parallel and perpendicular to the conducting surface. New experiments are under way looking at the three-dimensional flow structure and the plume dynamics near the thermal boundary layer. Finally, we remark that because the LSC has a preferred direction as mentioned in Sec. II (C), measurements in a levelled cell in fact give the same results as the ones obtained in a tilted cell presented in this paper.

\section{ACKNOWLEDGMENTS}

We gratefully acknowledge support of this work by the Research Grants Council of Hong Kong SAR under Projects No. CUHK403003 (C.S. and K.-Q.X.) and No. HKUST603003 (P.T.).
[1] R. Krishnamurti and L. N. Howard, Proc. Natl. Acad. Sci. U.S.A. 78, 1981 (1981).

[2] B. Castaing, G. Gnuaratne, F. Heslot, L. Kadanoff, A. Libchaber, S. Thomae, X.-Z. Wu, S. Zaleski, and G. Zanetti, J. Fluid Mech. 204, 1 (1989).

[3] A. Tilgner, A. Belmonte, and A. Libchaber, Phys. Rev. E 47, R2253 (1993).

[4] R. M. Kerr, J. Fluid Mech. 310, 139 (1996).

[5] Y.-B. Xin, K.-Q. Xia, and P. Tong, Phys. Rev. Lett. 77, 1266 (1996).

[6] T. Takeshita, T. Segawa, J. A. Glazier, and M. Sano, Phys. Rev. Lett. 76, 1465 (1996).

[7] S. Cioni, S. Ciliberto, and J. Sommeria, J. Fluid Mech. 335,
111 (1997).

[8] X.-L. Qiu and K.-Q. Xia, Phys. Rev. E 58, 486 (1998).

[9] X.-L. Qiu and K.-Q. Xia, Phys. Rev. E 58, 5816 (1998).

[10] R. Verzicco and R. Camussi, J. Fluid Mech. 383, 55 (1999).

[11] S. Grossmann and D. Lohse, J. Fluid Mech. 407, 27 (2000).

[12] X. Chavanne, F. Chillà, B. Chabaud, B. Castaing, and B. Hébral, Phys. Fluids 13, 1300 (2001).

[13] Z. A. Daya and R. E. Ecke, Phys. Rev. Lett. 87, 184501 (2001).

[14] X.-L. Qiu and P. Tong, Phys. Rev. E 64, 036304 (2001).

[15] J. J. Niemela, L. Skrbek, K. R. Sreenivasan, and R. J. Donnelly, J. Fluid Mech. 449, 169 (2001).

[16] K. R. Sreenivasan, A. Bershadskii, and J. J. Niemela, Phys. 
Rev. E 65, 056306 (2002).

[17] S. Lam, X.-D. Shang, S.-Q. Zhou, and K.-Q. Xia, Phys. Rev. E 65, 066306 (2002).

[18] S. Grossmann and D. Lohse, Phys. Rev. E 66, 016305 (2002).

[19] S. Grossmann and D. Lohse, J. Fluid Mech. 486, 105 (2003).

[20] J. J. Niemela and K. R. Sreenivasan, Europhys. Lett. 62, 829 (2003).

[21] E. Brown, A. Nikolaenko, and G. Ahlers Phys. Rev. Lett. (to be published).

[22] L. Kadanoff, in Proceedings of the Fifth EPS Liquid State Conference, Moscow, IPM USSR AS, 1990, Vol. 32, p. 596.

[23] L. Kadanoff, Phys. Today 54(8), 34 (2001).

[24] G. Zocchi, E. Moses, and A. Libchaber, Physica A 166, 387 (1990).

[25] H.-D. Xi, S. Lam, and K.-Q. Xia, J. Fluid Mech. 503, 47 (2004).

[26] M. Sano, X.-Z. Wu, and A. Libchaber, Phys. Rev. A 40, 6421 (1989).

[27] S. Ashkenazi and V. Steinberg, Phys. Rev. Lett. 83, 3641 (1999).

[28] X.-L. Qiu, S.-H. Yao, and P. Tong, Phys. Rev. E 61, R6075 (2000).

[29] X.-D. Shang and K.-Q. Xia, Phys. Rev. E 64, 065301(R) (2001).

[30] E. Villermaux, Phys. Rev. Lett. 75, 4618 (1995).

[31] X.-L. Qiu and P. Tong, Phys. Rev. Lett. 87, 094501 (2001).

[32] X.-L. Qiu and P. Tong, Phys. Rev. E 66, 026308 (2002).

[33] X.-L. Qiu, X.-D. Shang, P. Tong, and K.-Q. Xia, Phys. Fluids
16, 412 (2004).

[34] D. Funfschilling and G. Ahlers, Phys. Rev. Lett. 92, 194502 (2004).

[35] T. Haramina and A. Tilgner, Phys. Rev. E 69, 056306 (2004).

[36] R. J. Adrian, Annu. Rev. Fluid Mech. 23, 261 (1991).

[37] K.-Q. Xia, C. Sun, and S.-Q. Zhou, Phys. Rev. E 68, 066303 (2003).

[38] M. Raffel, C. Willert, and J. Kompenhans, Particle Image Velocimetry (Springer-Verlag, Berlin, 1998).

[39] S. Ciliberto, S. Cioni, and C. Laroche, Phys. Rev. E 54, R5901 (1996).

[40] F. Chillà, M. Rastello, S. Chaumat, and B. Castaing, Eur. Phys. J. B 40, 223 (2004).

[41] C. Sun, H.-D. Xi, and K.-Q. Xia Phys. Rev. Lett. (to be published).

[42] Y.-B. Xin and K.-Q. Xia, Phys. Rev. E 56, 3010 (1997).

[43] K.-Q. Xia, S. Lam, and S.-Q. Zhou, Phys. Rev. Lett. 88, 064501 (2002)

[44] S.-L. Lui and K.-Q. Xia, Phys. Rev. E 57, 5494 (1998).

[45] These movies are made by concatenating the consecutively measured instantaneous velocity field and they can be views at www.phy.cuhk.edu.hk/turbulence/movies/.

[46] X.-D. Shang, X.-L. Qiu, P. Tong, and K.-Q. Xia, Phys. Rev. Lett. 90, 074501 (2003).

[47] X.-D. Shang, X.-L. Qiu, P. Tong, and K.-Q. Xia, Phys. Rev. E 70, 026308 (2004).

[48] A. Parodi, J. von Hardenberg, G. Passoni, A. Provenzale, and E. A. Spiegel, Phys. Rev. Lett. 92, 194503 (2004). 